\title{
Alternative Representation of the Stability Condition for the Particle Swarm Optimization Algorithm
}

\author{
Yuji Wakasa, Kanya Tanaka, and Yuki Nishimura \\ Graduate School of Science and Engineering, Yamaguchi University \\ 2-16-1 Tokiwadai, Ube 755-8611, Japan \\ E-mail: wakasa@yamaguchi-u.ac.jp
}

\begin{abstract}
In the previous work, the stability condition of the particle swarm optimization algorithm has been derived by linear matrix inequality techniques. This paper provides an alternative representation of the stability condition, which can be checked more shortly and accurately than that in the previous work. The stability condition is described by nonlinear scalar inequalities. Also, we present a condition related to a decay rate of the particle swarm optimization algorithm in terms of nonlinear scalar inequalities. Numerical experiments are given to show that the largest lower bound of the decay rate can be used as a measure of convergence speed of the particle swarm optimization algorithm.
\end{abstract}

\section{Introduction}

Particle swarm optimization (PSO) is a kind of stochastic optimization, which is based on swarm intelligence such as bird flocking and fish schooling [1]. Recently, the PSO has been applied to many nonconvex optimization problems $[2,3,4]$ because it is not only effective to complicated problems but also easy to implement.

The PSO algorithm is described as a simple dynamical system with random variables, and therefore, we can analyze the behavior of the PSO algorithm by control theoretic approaches $[5,6,7,8]$. Trelea has showed a convergence condition of the PSO algorithm when the random variables are assumed to be constant, which leads to the limitations of the results [6]. Kadirkamanathan et al. have relaxed the assumption on the random variables, and have shown a different convergence condition under an equality constraint involving the random variables [8]. However, the result is still far from the exact behavior of the PSO algorithm. While these approaches are analytical, Yasuda et al. have shown a numerical approach based on many numerical experiments [9], which gives a more realistic convergence condition.

On the other hand, Wakasa et al. [10] have expressed the PSO algorithm as a system with multiplicative noise, and have derived its stability condition in terms of a linear matrix inequality (LMI). Unlike $[6,8]$, this analytical approach does not impose any assumptions on the random variables in the PSO algorithm. Consequently, it has been shown that the obtained analytical result is almost the same as the numerical result in [9]. The analytical method in [10] has an advantage that convergence of the PSO algorithm can be more easily checked for various setting parameters in the PSO algorithm than the method based on numerical experiments in [9]. Moreover, a measure for the decay rate of the PSO algorithm has been introduced, and a method for finding the largest lower bound on the decay rate has been presented in [10]. These results [10] are based on the LMI approach [11].

Although the LMI is efficiently solvable by convex optimization tools, a scalar condition is desirable to test it shortly and accurately. In this paper, we provide an alternative representation of the stability condition for the PSO algorithm based on the Lyapunov stability theorem. The stability condition is described by nonlinear scalar inequalities. Also, we present a condition related to the decay rate of the PSO algorithm in terms of nonlinear scalar inequalities. In numerical experiments, we focus on the verification that the largest lower bound of the decay rate can be used as a measure of the convergence speed of the PSO algorithm.

This paper is organized as follows. In Section 2, we briefly describe the result of stability analysis for systems with multiplicative noise via LMI techniques. In Section 3, we address the basic PSO algorithm, and show the main results in this paper. Then, Section 4 provides numerical experiments, and finally, Section 5 concludes the paper.

\section{Preliminaries}

In this section, we briefly provide the results on stability and decay rate analysis of systems with multiplicative noise [11]. The results are applied to analysis of the PSO algorithm in the next section.

We consider the discrete-time stochastic system

$$
\xi_{k+1}=\left(A+\sum_{i=1}^{L} A_{i} \theta_{i, k}\right) \xi_{k},
$$

where $\xi_{k} \in \Re^{n_{\xi}}$ is the state, $A, A_{1}, \ldots, A_{L} \in \Re^{n_{\varepsilon} \times n_{\xi}}$ 
are the coefficient matrices, and $\theta_{k}:=\left[\theta_{1, k}, \ldots, \theta_{L, k}\right]^{T}$ is a random variable. Denoting the expectation by $\mathbb{E}$, we assume that $\theta_{0}, \theta_{1}, \ldots$ are independent identically distributed random variables with

$$
\begin{aligned}
& \mathbf{E} \theta_{k}=0, \\
& \mathbf{E} \theta_{k} \theta_{k}^{T}=\operatorname{diag}\left(\sigma_{1}^{2}, \ldots, \sigma_{L}^{2}\right),
\end{aligned}
$$

where, for $i=1, \ldots, L, \sigma_{i}^{2}$ denotes the variance of $\theta_{i, k}$. We also assume that $\xi_{0}$ is independent of the process $\theta_{k}$.

For the state $\xi_{k}$, we define the state covariance matrix as

$$
M_{k}:=\mathbf{E} \xi_{k} \xi_{k}^{T} \text {. }
$$

From (1), we see that $M_{k}$ satisfies the linear recursion

$$
\begin{aligned}
& M_{k+1}=A M_{k} A^{T}+\sum_{i=1}^{L} \sigma_{i}^{2} A_{i} M_{k} A_{i}^{T} \\
& M_{0}=\mathbf{E} \xi_{0} \xi_{0}^{T} .
\end{aligned}
$$

If this linear recursion is stable, i.e., regardless of $\xi_{0}$, $\lim _{k \rightarrow \infty} M_{k}=0$, we say the system is mean-square stable. Mean-square stability implies, for example, that $\xi_{k} \rightarrow 0$ almost surely. The following theorem holds for system (1) [11].

Theorem 1 System (1) is mean-square stable if and only if, for any given $Q>0$, there exists a matrix $P>0$ satisfying the linear matrix equality:

$$
A^{T} P A-P+\sum_{i=1}^{L} \sigma_{i}^{2} A_{i}^{T} P A_{i}+Q=0 .
$$

Although the condition in Theorem 1 is expressed as a linear matrix equation, it is easily seen that the equivalent condition can be written as the existence of $P>0$ satisfying the LMI

$$
A^{T} P A-P+\sum_{i=1}^{L} \sigma_{i}^{2} A_{i}^{T} P A_{i}<0 .
$$

Theorem 1 is derived by applying the Lyapunov stability theorem to system (2) as shown in [11].

Next, we consider a decay rate $\nu[11,12]$ for system (1). The decay rate is defined as the largest $\nu>1$ such that $\lim _{k \rightarrow \infty} \nu^{k}\left(\mathbf{E} \xi_{k} \xi_{k}^{T}\right)=0$. After the same discussion as in $[11,12]$, we can obtain the following theorem.

Theorem $2 \tilde{\nu}=1 / \mu$ is a lower bound of the decay rate for system (1) if and only if, for any given $Q>0$, there exists a matrix $P>0$ satisfying the linear matrix equality:

$$
A^{T} P A-\mu P+\sum_{i=1}^{L} \sigma_{i}^{2} A_{i}^{T} P A_{i}+Q=0 .
$$

As in the case of Theorem 1, the equality condition in Theorem 2 can be equivalently written in terms of an LMI [10]. Then, we can compute the largest lower bound of the decay rate by applying a bisection algorithm for $\mu$ and checking whether there is a matrix $P>0$ satisfying the LMI as described in [10].

\section{Stability and Decay Rate Analysis}

\subsection{Basic PSO algorithm}

The standard PSO algorithm is applied to an unconstrained minimization problem. In general, a swarm consists of multiple particles, and each particle position is a potential solution to the optimization problem in $n$-dimensional space. According to an update rule, each particle position is updated, and its previous best position and the best position among all particles are stored. Although each dimension of a particle position vector is influenced by the other dimensions through the function evaluation, we analyze the particle behavior approximately by assuming that each dimension of a particle position is updated independently of the others. The basic update rule of the PSO algorithm in one dimension is given by

$$
\begin{aligned}
v_{k+1} & =\alpha v_{k}+\beta_{k}^{(p)}\left(x_{k}^{(p)}-x_{k}\right)+\beta_{k}^{(g)}\left(x_{k}^{(g)}-x_{k}\right) \\
x_{k+1} & =x_{k}+v_{k+1}
\end{aligned}
$$

where $v_{k}$ is the particle velocity at the $k$ th iteration, $x_{k}$ is the particle position at the $k$ th iteration, $x_{k}^{(p)}$ is the personal best position achieved up to the $k$ th iteration, $x_{k}^{(g)}$ is the best global position among all particles, $\alpha$ is the inertia factor, and $\beta_{k}^{(p)} \sim \mathcal{U}\left[0, c^{(p)}\right]$ and $\beta_{k}^{(g)} \sim \mathcal{U}\left[0, c^{(g)}\right]$ are random variables according to uniform distributions with constants $c^{(p)}$ and $c^{(g)}$.

\subsection{Main results}

Now we introduce the following 2-dimensional vectors:

$$
\begin{gathered}
\xi_{k+1}:=\left[\begin{array}{c}
x_{k} \\
x_{k+1}
\end{array}\right], \\
w_{k}:=\left[\begin{array}{c}
x_{k}^{(p)} \\
x_{k}^{(g)}
\end{array}\right] .
\end{gathered}
$$

Using these vectors, we can express the PSO dynamics as follows:

$$
\begin{aligned}
\xi_{k+1}= & {\left[\begin{array}{cc}
0 & 1 \\
-\alpha & 1+\alpha-\beta_{k}^{(p)}-\beta_{k}^{(g)}
\end{array}\right] \xi_{k} } \\
& +\left[\begin{array}{cc}
0 & 0 \\
\beta_{k}^{(p)} & \beta_{k}^{(g)}
\end{array}\right] w_{k} .
\end{aligned}
$$

System (5) is a system whose coefficient matrices contain random variables. To analyze system (5) more easily, we also express the random variables $\beta_{k}^{(p)}, \beta_{k}^{(g)}$ as

$$
\begin{aligned}
& \beta_{k}^{(p)}=c^{(p)} \theta_{1, k}+\frac{c^{(p)}}{2} \\
& \beta_{k}^{(g)}=c^{(g)} \theta_{2, k}+\frac{c^{(g)}}{2},
\end{aligned}
$$

where $\theta_{1, k}, \theta_{2, k} \sim \mathcal{U}[-1 / 2,1 / 2]$ are both random variables with the uniform distribution. Here system (5) is 
represented by

$$
\xi_{k+1}=A \xi_{k}+B w_{k}+\sum_{i=1}^{2}\left(A_{i} \xi_{k}+B_{i} w_{k}\right) \theta_{i, k}
$$

where

$$
\begin{aligned}
& A=\left[\begin{array}{cc}
0 & 1 \\
-\alpha & 1+\alpha-\frac{c^{(p)}}{2}-\frac{c^{(g)}}{2}
\end{array}\right], \\
& B=\left[\begin{array}{cc}
0 & 0 \\
\frac{c^{(p)}}{2} & \frac{c^{(g)}}{2}
\end{array}\right], \\
& A_{1}=\left[\begin{array}{cc}
0 & 0 \\
0 & -c^{(p)}
\end{array}\right], A_{2}=\left[\begin{array}{cc}
0 & 0 \\
0 & -c^{(g)}
\end{array}\right], \\
& B_{1}=\left[\begin{array}{cc}
0 & 0 \\
c^{(p)} & 0
\end{array}\right], B_{2}=\left[\begin{array}{cc}
0 & 0 \\
0 & c^{(g)}
\end{array}\right] .
\end{aligned}
$$

System (6) is a form of systems with multiplicative noise. In the PSO algorithm, a solution is searched by the interaction among particles through the update of $x_{k}^{(p)}$ and $x_{k}^{(g)}$. In this paper, however, we assume that these variables $x_{k}^{(p)}$ and $x_{k}^{(g)}$ are constant for ease of analysis, which means that $w_{k}$ is a constant exogenous input. Therefore, we can apply the results in Section 2 to system (6) to analyze the stability and decay rate.

Also, we may regard $\theta_{k}:=\left[\theta_{1, k}, \theta_{2, k}\right]^{T}$ as an independent, identically distributed random variable with

$$
\begin{aligned}
& \mathrm{E} \theta_{k}=0, \\
& \mathrm{E} \theta_{k} \theta_{k}^{T}=\operatorname{diag}(1 / 12,1 / 12) .
\end{aligned}
$$

Then we obtain the following theorem on the stability of the PSO algorithm by applying Theorem 1 to system (6).

Theorem 3 System (6) is mean-square stable if and only if, for any given $Q>0$, there exists a matrix $P>0$ satisfying the linear matrix equality:

$$
A^{T} P A-P+\sum_{i=1}^{2} \frac{1}{12} A_{i}^{T} P A_{i}+Q=0 .
$$

Although the condition in Theorem 3 is expressed by a matrix equality, we can obtain an alternative representation as follows.

Theorem 4 System (6) is mean-square stable if and only if there exist $\alpha, c^{(p)}$ and $c^{(g)}$ satisfying the following nonlinear scalar inequalities:

$$
\begin{aligned}
& (\alpha+1)^{2}\left(12 \alpha^{2}+\left(c^{(p)}\right)^{2}+\left(c^{(g)}\right)^{2}-12\right) \\
& -3\left(\alpha^{2}-1\right)\left(2 \alpha-c^{(p)}-c^{(g)}+2\right)^{2}<0 \\
& (\alpha+1)^{2}\left(-12 \alpha^{2}+\left(c^{(p)}\right)^{2}+\left(c^{(g)}\right)^{2}-12\right) \\
& +3\left(\alpha^{2}+1\right)\left(2 \alpha-c^{(p)}-c^{(g)}+2\right)^{2}<0
\end{aligned}
$$

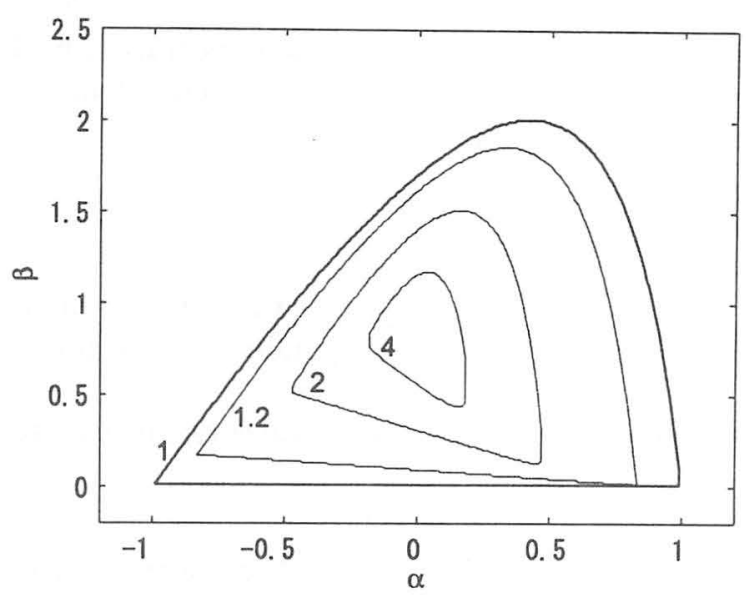

Fig. 1: Contour plots of the largest lower bounds of the decay rate when $\beta=c^{(p)}=c^{(g)}$.

Sketch of the Proof: We set $Q=I$ and

$$
P=\left[\begin{array}{ll}
p_{1} & p_{2} \\
p_{2} & p_{3}
\end{array}\right]
$$

and solve the linear matrix equality (7) with respect to $P$. Then, using the relationship that $P$ is positive definite if and only if

$$
p_{1}>0, \quad p_{1} p_{3}-p_{2}^{2}>0,
$$

we derive two scalar inequalities. Arranging these inequalities, we obtain (8).

Although the LMI conditions derived in [10] can be checked with reasonable time and accuracy by using a recently developed convex optimization tool on a computer, the scalar conditions shown in this paper can be checked more shortly and accurately.

Next, we consider the decay rate of system (6) to evaluate the convergence speed of the PSO algorithm. We obtain the following theorem by applying Theorem 2 to system (6).

Theorem $5 \tilde{\nu}=1 / \mu$ is a lower bound of the decay rate for system (6) if and only if, for any given $Q>0$, there exists a matrix $P>0$ satisfying the linear matrix equality:

$$
A^{T} P A-\mu P+\sum_{i=1}^{2} \frac{1}{12} A_{i}^{T} P A_{i}+Q=0 .
$$

In a similar way to the stability condition, we obtain an alternative representation of the condition regarding the decay rate.

Theorem $6 \tilde{\nu}=1 / \mu$ is a lower bound of the decay rate for system (6) if and only if there exist $\alpha, c^{(p)}$ and $c^{(g)}$ 


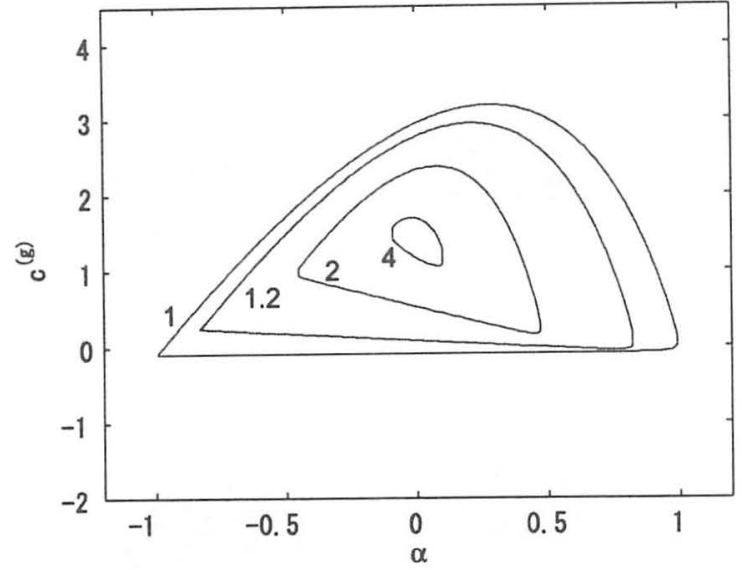

(a) $c^{(p)}=0.1$

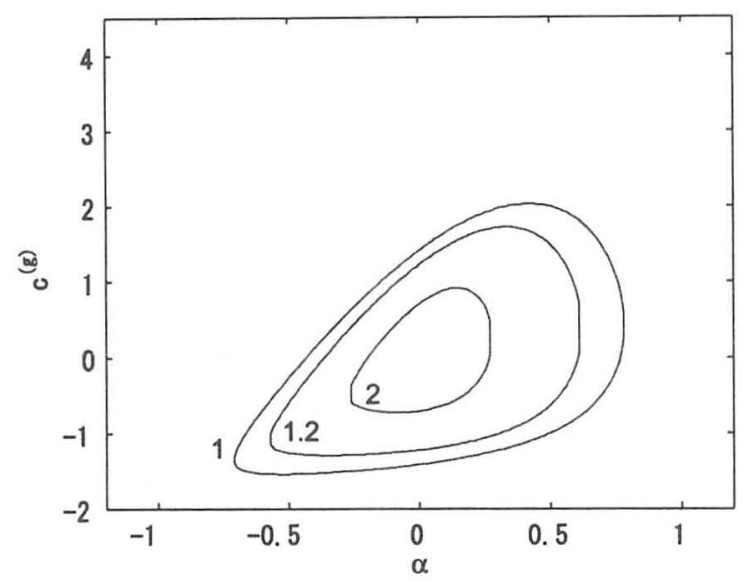

(c) $c^{(p)}=2$

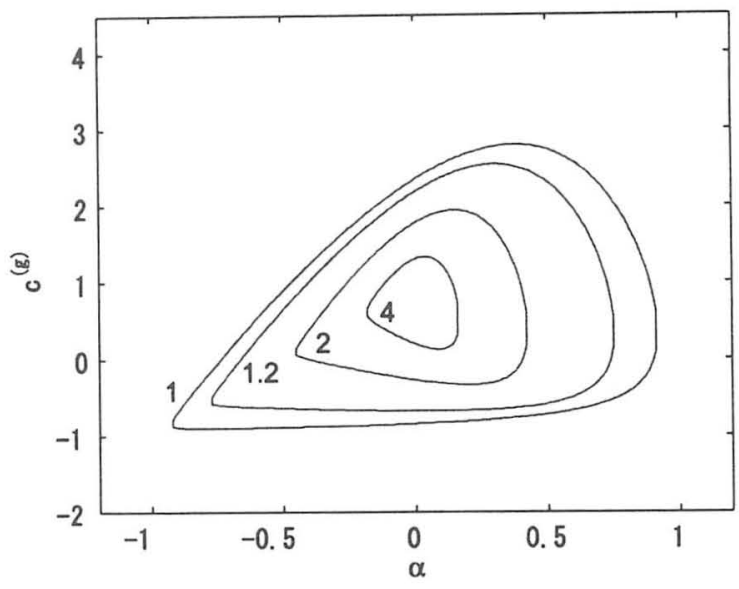

(b) $c^{(p)}=1$

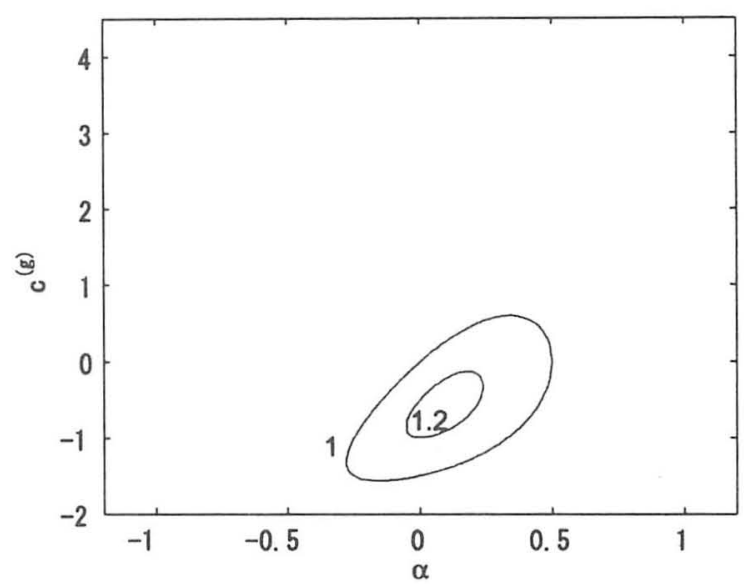

(d) $c^{(p)}=3$

Fig. 2: Contour plots of the largest lower bounds of the decay rate when $c^{(p)}=0.1,1,2,3$.

satisfying the following nonlinear scalar inequalities:

$$
\begin{aligned}
& (\alpha+\mu)^{2}\left(12 \alpha^{2}+\mu\left(\left(c^{(p)}\right)^{2}+\left(c^{(g)}\right)^{2}\right)-12 \mu^{2}\right) \\
& -3 \mu\left(\alpha^{2}-\mu^{2}\right)\left(2 \alpha-c^{(p)}-c^{(g)}+2\right)^{2}<0, \quad(10 \mathrm{a}) \\
& (\alpha+\mu)^{2}\left(-12 \alpha^{2}+\mu^{2}\left(\left(c^{(p)}\right)^{2}+\left(c^{(g)}\right)^{2}\right)-12 \mu^{3}\right) \\
& +3 \mu\left(\alpha^{2}+\mu^{3}\right)\left(2 \alpha-c^{(p)}-c^{(g)}+2\right)^{2}<0 . \quad(10 \mathrm{~b})
\end{aligned}
$$

We can compute the largest lower bound $\tilde{\nu}_{\max }$ of the decay rate by applying a bisection algorithm for $\mu$ and checking whether the two inequalities (10) are both satisfied.

Fig. 1 shows the contour plots of the largest lower bounds $\tilde{\nu}_{\max }$ of the decay rate that are computed based on Theorem 6, where $c^{(p)}$ and $c^{(g)}$ are equal, i.e., $\beta=$ $c^{(p)}=c^{(g)}$. Note that the interior region formed with $\tilde{\nu}_{\max }=1$ corresponds to the stability region because (10) with $\tilde{\nu}=1 / \mu=1$ is consistent with (8).

Fig. 2 shows the contour plots of the largest lower bounds $\tilde{\nu}_{\max }$ for the case of $c^{(p)} \neq c^{(g)}$ where the vertical axis is $c^{(g)}$, and $c^{(p)}$ is fixed as $0.1,1,2$, and 3 . Note that the parameter regions with the largest lower bounds are symmetric for $c^{(p)}$ and $c^{(g)}$ because the left hand sides of (10) are unchanged even when $c^{(p)}$ and $c^{(g)}$ are exchanged each other.

\section{Numerical Experiments}

The validity of the stability condition in Theorem 3 has been verified by comparing the results in [10] and [9]. In this section, we focus on the verification of the decay rate condition in Theorems 5 and 6 .

We consider a $17 \times 17$ grid in $[-1,1] \times[0,2]$ in the parameters region of $\alpha$ and $\beta=c^{(p)}=c^{(g)}$, and examine convergence time of the PSO algorithm with each parameter set in the stability region. Four kinds of functions to be minimized are used as test functions [6]. The PSO algorithm is performed 100 times for each parameter set and each objective function.

As shown in Fig. 3, the convergence time $k_{c}$ is defined as the time when the mean of the best function value 


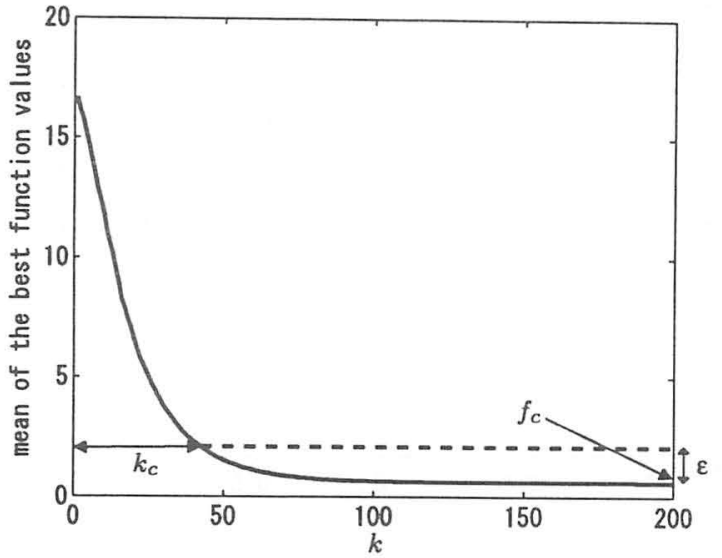

Fig. 3: Definition of the convergence time.

reaches $f_{c}+\varepsilon$ where $f_{c}$ and $\varepsilon$ denote the mean of the best function values after $k=200$ and a specified tolerance, respectively.

The test functions are as follows.

$$
\begin{aligned}
f_{\text {Sphere }} & =\sum_{i=1}^{n} x_{i}^{2} \\
f_{\text {Rosenbrock }} & =\sum_{i=1}^{n-1}\left(100\left(x_{i+1}-x_{i}^{2}\right)^{2}+\left(x_{i}-1\right)^{2}\right), \\
f_{\text {Rastrigin }} & =\sum_{i=1}^{n}\left(x_{i}^{2}-10 \cos \left(2 \pi x_{i}\right)+10\right) \\
f_{\text {Griewank }} & =\frac{1}{4000} \sum_{i=1}^{n} x_{i}^{2}-\prod_{i=1}^{n} \cos \left(\frac{x_{i}}{\sqrt{i}}\right)+1
\end{aligned}
$$

Fig. 4 shows the shapes of the functions in 2dimensional space. The optimal values of the four functions are all 0 achieved at $x_{i}=1$ for $f_{\text {Rosenbrock }}$ and at the origin for the other functions.

We consider a minimization problem for each function in 5-dimensional space. Let the number of particles be 10 , and the maximum number of iterations be 200. Initial particle positions are randomly given from $\mathcal{U}[-5,5]^{5}$.

Under the above settings, we performed the PSO algorithm 100 times for each function and each parameter set. Figs. 5-8 show the distributions of the convergence time for the test functions. We see from the figures that the experimental distributions are similar to the contour plots of $\tilde{\nu}_{\max }$ in Fig. 1.

\section{Conclusion}

In this paper, we have provided alternative representations of the stability and decay rate conditions for the PSO algorithm, which can be computed shortly and accurately. We have provided numerical experiments to

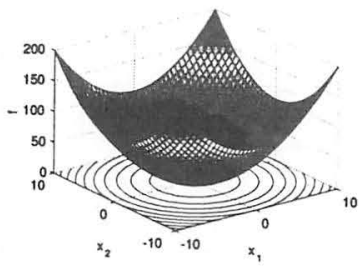

(a) $f_{\text {Sphere }}$

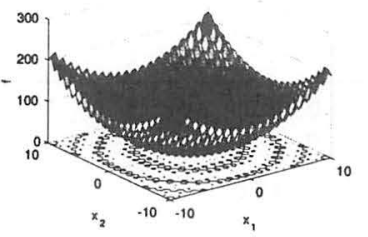

(c) $f_{\text {Rastrigin }}$

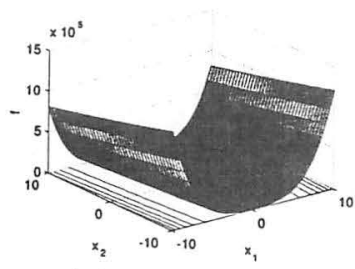

(b) $f_{\text {Rosenbrock }}$

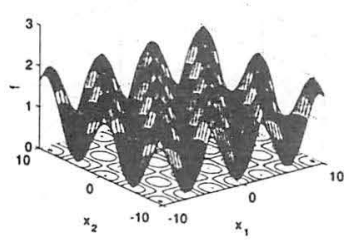

(d) $f_{\text {Griewank }}$
Fig. 4: Shapes of the test functions.

show that the largest lower bound of the decay rate can be used as a measure of convergence speed of the PSO algorithm.

In general, there is a tradeoff between exploitation and exploration abilities of this type of optimization algorithm. Therefore, it is important to analyze the exploration ability of the PSO algorithm as well as the exploitation ability presented in this paper, which is one of the future research directions.

\section{References}

[1] J. Kennedy and R. Eberhart: Particle swarm optimization, Proc. 1995 IEEE International Conference on Neural Networks, pp. 1942-1948, 1995.

[2] H. Yoshida, K. Kawata, Y. Fukuyama, S. Takayama, and Y. Nakanishi: A particle swarm optimization for reactive power and voltage control considering voltage security assessment, IEEE Trans. Power Systems, vol. 15, no. 4, pp. 12321239, 2000.

[3] T. Kim, I. Maruta, and T. Sugie: Robust PID controller tuning based on the constrained particle swarm optimization, Automatica, vol. 44, pp. 1104-1110, 2008.

[4] I. Maruta, T. H. Kim, and T. Sugie: Fixedstructure $H_{\infty}$ controller synthesis: A metaheuristic approach using simple constrained particle swarm optimization, Automatica, vol. 45, pp. $553-559,2009$

[5] M. Clerc and J. Kennedy: The particle swarmExposition, stability, and convergence in a multidimensional complex space, IEEE Trans. Evolutionary Computation, vol. 6, no. 1, pp. 58-73, 2002. 
[6] I. C. Trelea: The particle swarm optimization algorithm: convergence analysis and parameter selection, Information Processing Letters, vol. 85, pp. 317-325, 2003.

[7] H. M. Emara and H. A. A. Fattah: Continuous swarm optimization technique with stability analysis, Proc. 2004 American Control Conference, pp. 2811-2817, 2004.

[8] V. Kadirkamanathan, K. Selvarajah, and P. J. Fleming: Stability analysis of the particle dynamics in particle swarm optimizer, IEEE Trans. Evolutionary Computation, vol. 10, no. 3, pp. 245-255, 2006.

[9] K. Yasuda, N. Iwasaki, G. Ueno, and E. Aiyoshi: Particle swarm optimization: a numerical stability analysis and parameter adjustment based on swarm activity, IEEJ Trans. Electrical and Electronic Engineering, vol. 3, pp. 642-659, 2008.

[10] Y. Wakasa, Y. Watanabe, A. Iwamoto, K. Tanaka, and T. Akashi: Stability analysis of the particle swarm optimization algorithm, Proc. 40th ISCIE International Symposium on Stochastic Systems Theory and Its Applications, pp. 131-136, 2009.

[11] S. Boyd, L. El Ghaoui, E. Feron, and V. Balakrishnan: Linear Matrix Inequalities in System and Control, SIAM, 1994.

[12] L. Xiao, A. Hassibi, and J. How: Control with random communication delays via a discrete-time jump system approach, Proc. 2000 American Control Conference, vol. 3, pp. 2199-2204, 2000.

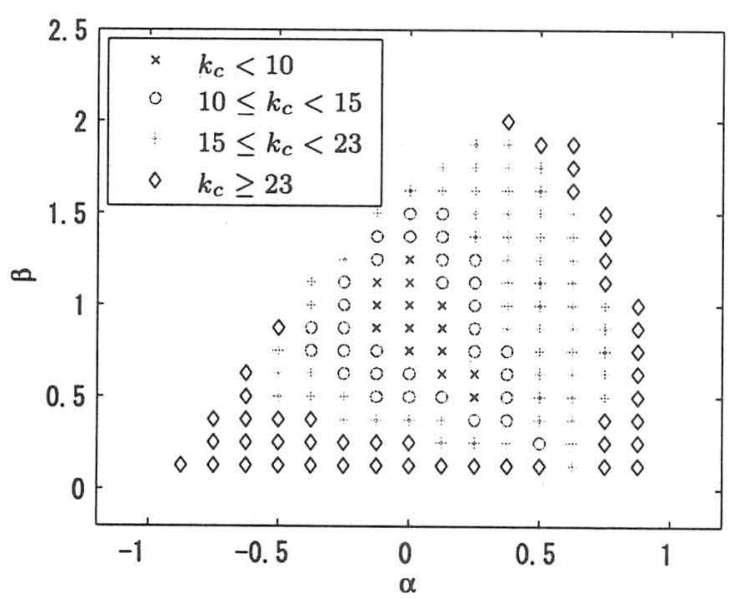

Fig. 5: Distribution of the convergence time for $f_{\text {Sphere }}$ where $\varepsilon=0.1$.

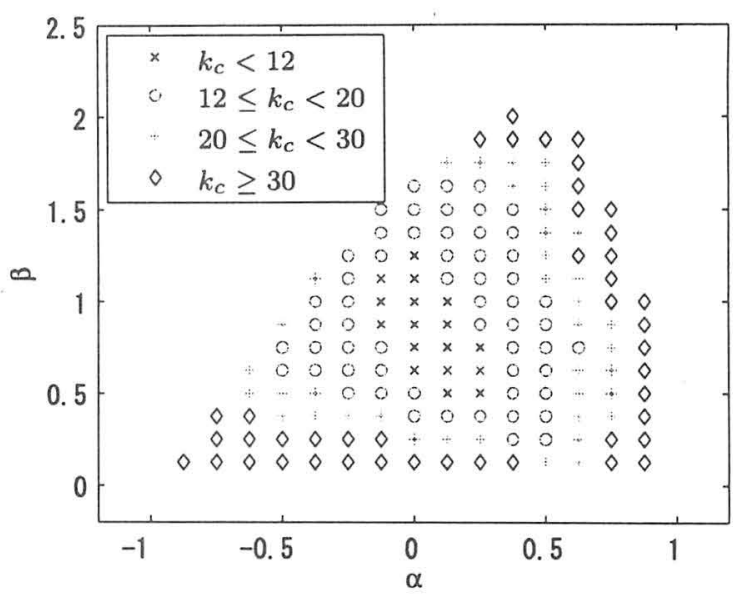

Fig. 6: Distribution of the convergence time for $f_{\text {Rosenbrock }}$ where $\varepsilon=10$.

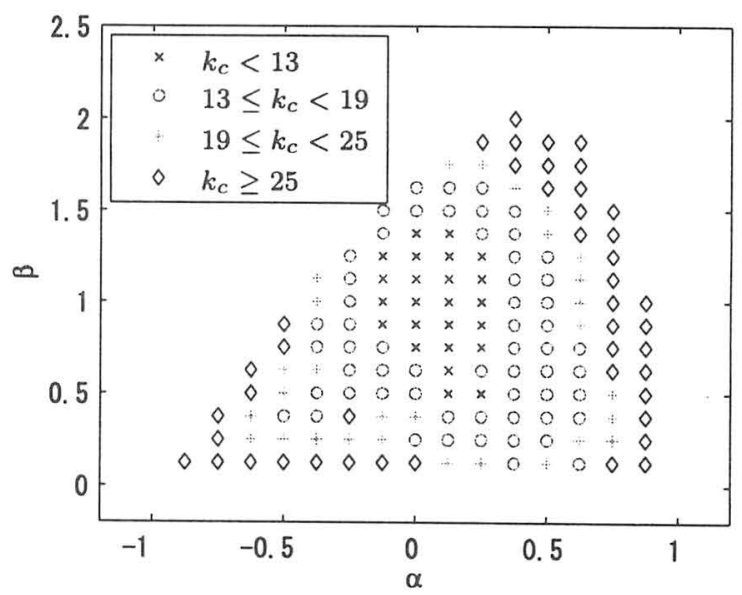

Fig. 7: Distribution of the convergence time for $f_{\text {Rastrigin }}$ where $\varepsilon=5$.

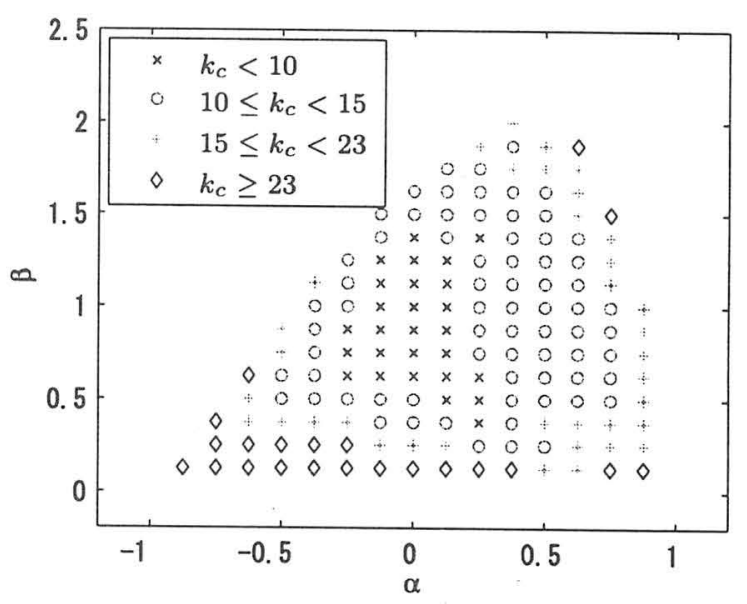

Fig. 8: Distribution of the convergence time for $f_{\text {Griewank }}$ where $\varepsilon=0.1$. 